\title{
MINIMUM VARIANCE REINSURANCE *)
}

BY

\section{STEFAN VAJDA}

London

In a paper entitled "An Attempt to Determine the Optimum Amount of Stop Loss Reinsurance" (XVIth Int. Congr. Act. Bruxelles I960) Karl Borch has shown that, if the reinsurance premium is given, the smallest variance of the cedent's payments is obtained by a stop-loss reinsurance contract. Paul Markham Kahn, in "Some Remarks on a Recent Paper by Borch", a paper read to the I96r Astin Colloquium, has given an elegant proof of this theorem which appears to apply also to cases not considered by Borch. In this paper we study the problem from the reinsurer's point of view and it will be seen that, under natural conditions which are also used in the proof of the Borch-Kahn theorem, the minimum variance of the reinsurer's payments is obtained by a quota contract. This focusses attention on a peculiar opposition of interests of the two partners of a reinsurance contract. However, we do not enter any further into the investigation of a possible resolution of this conflict.

We study a problem concerning the division of risk between a cedent and his reinsurer. The risk may refer to a whole portfolio (in which case one might consider a Stop-Loss contract), or to a single contract (when an Excess-Loss contract is a possibility). We shall here use the nomenclature of a portfolio reinsurance.

Let it be assumed that a function $F(x)$ is known which gives the probability of a total claim not exceeding $x$. We have then in Stieltjes integral notation

$$
\int_{0}^{\infty} d F(x)=\mathbf{I}
$$

The two partners to a reinsurance arrangement agree that the reinsurer reimburses $m(x) \cdot x$ out of a claim of $x$, where $m(x)$ is a continuous and differentiable function of $x$ and $0 \leqslant m(x) \leqslant \mathrm{r}$.

*) Paper presented to the Rättvik Colloquium i96t. 
The net reinsurance premium is then

and its variance is

$$
\int_{0}^{\infty} m(x) \cdot x d F(x)=P
$$

$$
\int_{0}^{\infty}[m(x) \cdot x]^{2} d F(x)-P^{2}
$$

It would be absurd to demand that $m(x)$ should be chosen so as to minimize the variance, since this could be achieved trivially by refraining from underwriting altogether. However, we may ask for the minimum of the variance subject to a given amount of the reinsurance premium, the latter being different from zero on the one and from $\int_{0}^{\infty} x d F(x)$ on the other hand.

Alternatively, we may ask for the minimum of the coefficient of variation, whose square can be written

$$
\frac{\int_{0}^{\infty}[m(x) \cdot x]^{2} d F(x)}{\left[\int_{0}^{\infty} m(x) \cdot x d F(x)\right]^{2}}-\mathbf{I} \text {. }
$$

For the purpose of illustration, we mention the following possibilities:

(i) $m(x)=c$ (constant). This is a quota reinsurance contract. The square of the coefficient of variation is

$$
\frac{\int_{0}^{\infty} x^{2} d F(x)}{\left[\int_{0}^{\infty} x d F(x)\right]^{2}}-\mathrm{I}
$$

which, it will be noticed, is independent of $c$.

$$
\text { (ii) } \begin{aligned}
m(x) & =0 \text { for } 0 \leq \dot{x} \leq a \\
& =(x-a) / x \text { for } x \geqslant a .
\end{aligned}
$$

This is a stop-loss reinsurance. The coefficient of variation is

$$
\frac{\int_{0}^{\infty}(x-a)^{2} d F(x)}{\left[\int_{0}^{\infty}(x-a) d F(x)\right]^{2}}-\mathbf{I}
$$

and for $a=0$ this reduces to the formula of case (i). 
We now restrict the family of functions $m(x)$ which we want to consider even further, by stipulating that $m(x)$ be a non-decreasing function of $x$. This agrees with the spirit of reinsurance, at least in most cases. We shall prove that the coefficient of variation is smallest when $m(x)=c$ (i.e. in case (i) above).

The constant - I is irrelevant and we shall only deal with the fraction. Our argument is as follows:

Consider some function $m(x)$ and assume that, starting from a given value $t$, we replace $m(x)$ by the constant $m(t)$. We obtain

$$
\frac{\int_{0}^{t}[m(x) \cdot x]^{2} d F(x)+[m(t)]^{2} \int_{t}^{\infty} x^{2} d F(x)}{\left[\int_{0}^{t} m(x) \cdot x d F(x)+m(t) \cdot \int_{1}^{\infty} x d F(x)\right]^{2}} .
$$

If it can be shown that this expression does not decrease with increasing $t$, then it follows that it will be the smaller, the earlier we replace $m(x)$ by a constant $m(t)$, so that the expression is smallest when $m(x)$ is taken to be constant from $t=0$, i.e. $m(x)=m(0)$ for all $x$.

Let the natural logarithm of the last expression above be denoted by $J(t)$. It is sufficient to show that $J(t)$ does not decrease with increasing $t$, because then this is true of $\exp (J(t))$ as well. We have

$$
\left.\begin{array}{r}
\frac{d J(t)}{d t}=\frac{2 d m(t)}{d t}\left\{\frac{\int_{t}^{\infty} x^{2} d F(x)}{\left(\int_{0}^{t}[m(x) x]^{2} d F(x)+[m(t)]^{2} \int_{t}^{\infty} x^{2} d F(x)\right.}\right. \\
-\frac{\int_{t}^{\infty} x d F(x)}{\int_{0}^{t} m(x) x d F(x)+m(t) \int_{t}^{\infty} x d F(x)}
\end{array}\right\} .
$$

$d m(t) / d t$ was assumed to be non-negative, and it suffices therefore to show that the expression in brackets cannot be negative. Taking the reciprocals of the fractions, we have to prove that

$$
\frac{\int_{0}^{t}[m(x) x]^{2} d F(x)}{\int_{0}^{\infty} x^{2} d F(x)}+[m(t)]^{2} \leq \frac{\int_{0}^{t} m(x) \cdot x d F(x)}{\int_{1}^{\infty} x d F(x)}+m(t) .
$$


We have $[m(t)]^{2} \leq m(t)$, because $m(t) \leq \mathrm{I}$. For $t=\mathrm{I}$ the required result is immediate, because $m(x) . x$ in the numerator is then $\leqslant \mathrm{I}$ and its square is not larger than itself, while in the denominator $x^{2}$ is not smaller than $x$. (Note that the limits of integration are $o$ and $t$ in the numerator, and $t$ and $\infty$ in the denominator.

Introducing the new variable $y=x / t$, we transform thereby the limits $o, t$, and $\infty$ of integration into $0, \mathrm{I}$, and $\infty$. The fractions in the integrals remain the same as they were before, after cancellations. Our statement is thus proved. The result is independent of the form of $F(x)$.

The coefficient of variation is independent of the choice of the constant $m(0)=c$, as has been mentioned. The actual value of $c$ will depend on the choice of $P$. 